\title{
Моделирование активированного электрическим полем спекания термоэлектриков
}

\author{
() Л.П. Булат ${ }^{1}$, А.В. Новотельнова ${ }^{1}$, В.Б. Освенский ${ }^{2}$, А.С. Тукмакова $^{1, \uparrow}$, Д. Ережеп ${ }^{1}$ \\ ${ }^{1}$ Университет ИТМО, \\ 197101 Санкт-Петербург, Россия \\ ${ }^{2}$ ОАО „Гиредмет“, \\ 119017 Москва, Россия \\ ฯ E-mail: tukmashh@gmail.com
}

(Получена 12 декабря 2016 г. Принята к печати 19 декабря 2016 г.)

С использованием нестационарной компьютерной модели рассмотрен процесс активированного полем спекания составных ветвей термоэлементов. Предложена модификация оснастки. Оснастка асимметричной формы, содержащая изолирующий слой, способствует формированию в образце перепада температур, достигающего нескольких сотен градусов. Проанализировано влияние толщины электроизоляционного слоя на величину осевого и радиального перепадов температур в образцах. Показана возможность понижения радиального температурного градиента.

DOI: 10.21883/FTP.2017.06.44551.11

Эффективность термоэлектриков оценивается параметром безразмерной термоэлектрической добротности ZT:

$$
Z T=\frac{\sigma \alpha^{2}}{\kappa} T
$$

где $T$ - абсолютная температура, $\sigma$ - коэффициент электропроводности, $\kappa-$ коэффициент теплопроводности, $\alpha-$ коэффициент термоэдс. Значения $\sigma, \kappa$ и $\alpha$ являются функциями температуры. В процессе работы термоэлемента температура вдоль его ветвей изменяется. При использовании однородных термоэлектриков добротность также изменяется вдоль ветви термоэлемента $[1,2]$. Для повышения эффективности генераторных модулей применяются составные ветви из материалов различного химического состава или ветви с неоднородным распределением свойств [3-5]. Состав слоев подбирается таким образом, чтобы каждый имел максимальное значение $Z T$ при приходящейся на него рабочей температуре. Получение таких ветвей возможно из нанопорошков с применением активированного полем спекания (АПС) в градиентном температурном поле [6,7]. Метод АПС позволяет сохранить при компактировании наноразмерные зерна термоэлектриков и достичь значений безразмерной добротности спеченного материала $Z T>1[8,9]$.

Асимметричное расположение матрицы пресс-формы, обеспечивающее контакт матрицы пресс-формы с нижней графитовой вставкой, позволяет формировать в процессе спекания температурный перепад по высоте образца $\Delta T_{z} \quad[6,10]$. Перепад температур по высоте образца при этом составляет десятки градусов, что недостаточно для спекания слоев из материалов с большим расхождением температур спекания. В данной работе рассмотрен способ увеличения $\Delta T_{z}$ путем внесения электроизоляционного слоя в асимметричную оснастку.
Моделирование процесса АПС проводилось методом конечных элементов на примере спекания термоэлектриков в установке SPS-511S (Япония) [10]. Геометрическая модель установки с модифицированной оснасткой приведена на рис. 1.

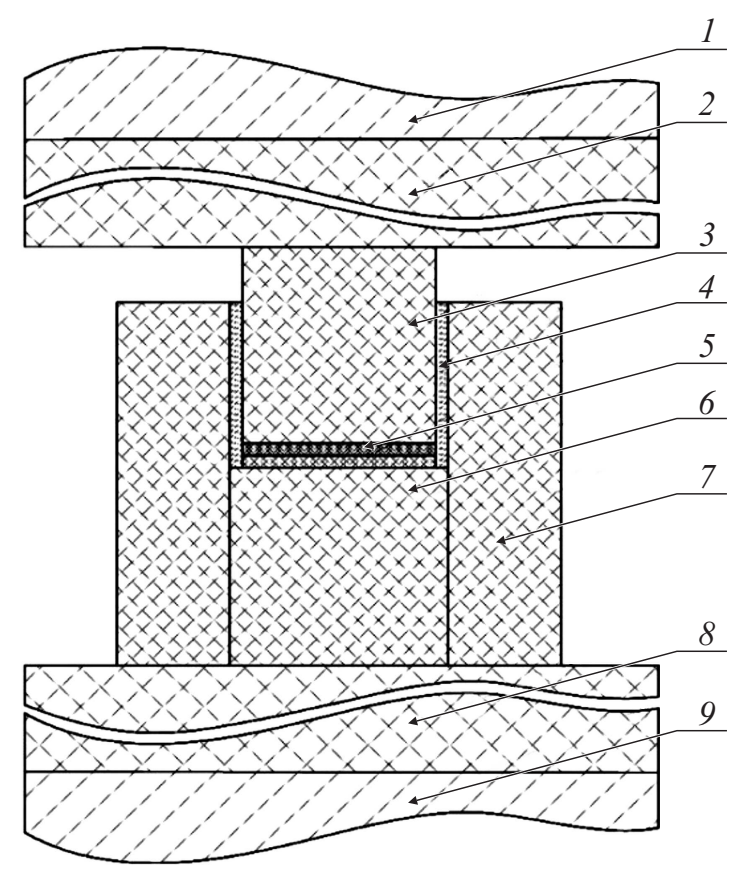

Рис. 1. Схема установки АПС с оснасткой, содержащей слой электрического изолятора и матрицу пресс-формы, контактирующую с нижней графитовой вставкой. 1,9- верхний и нижний стальные электроды (диаметр 80 мм, высота 60 мм); 2,8 - верхняя и нижняя графитовые вставки (диаметр 80 мм, высота 60 мм); 3, 6 - верхний и нижний графитовые пуансоны (диаметр 20 мм, высота 20 мм); 4 - изоляционный слой; 5 - образец; 7 - графитовая матрица пресс-формы (внутренний диаметр 20 мм, внешний диаметр 40 мм, высота 40 мм). 


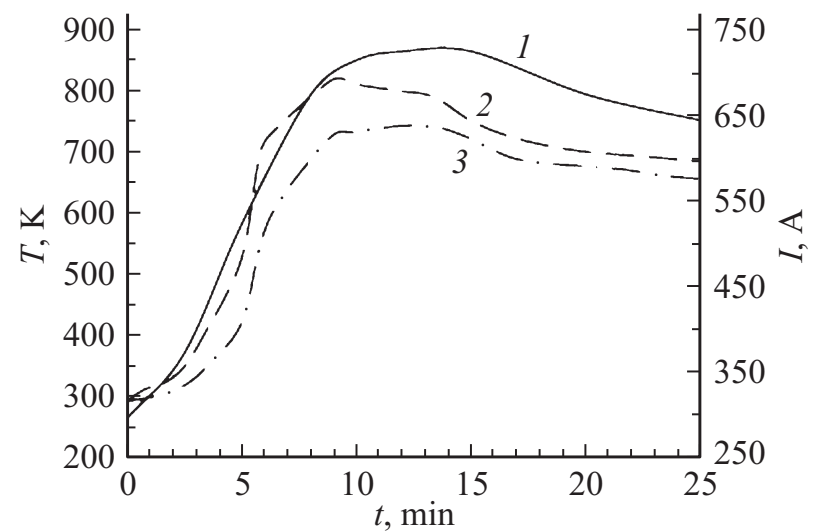

Рис. 2. Временна́я экспериментальная зависимость тока в ходе АПС процесса (1); расчетные временны́е зависимости температуры на верхней (2) и на нижней (3) поверхностях образца однородного состава при толщине изоляционного слоя $a=1 \mathrm{Mм}$.

Распределение электрического потенциала описывается дифференциальным уравнением

$$
\nabla \mathbf{J}=\nabla(\sigma \mathbf{E})=\nabla(-\sigma \nabla V)=0,
$$

где $\mathbf{J}=\sigma \mathbf{E}-$ плотность электрического тока, $\mathbf{E}=-\nabla V-$ напряженность электрического поля, $V-$ электрический потенциал.

Уравнение теплопроводности может быть записано в виде

$$
\rho c_{p} \frac{\partial \tau}{\partial t}=\nabla(\kappa \nabla T)+q_{j}
$$

где $c_{p}$ - теплоемкость при постоянном давлении, $\rho-$ плотность, $q_{j}=\mathbf{J} \cdot \mathbf{E}-$ теплота Джоуля.

В процессе АПС через верхний и нижний охлаждаемые водой электроды пропускается импульсный ток. Длительность импульса составляет 3.3 мс, скважность 2.2; за каждым пакетом из 12 импульсов следует 2 периода отключения тока. Амплитуда импульсов изменяется в процессе спекания, благодаря чему изменяется действующая плотность тока. Экспериментальная зависимость действующего тока $I$ от времени $t$ представлена на рис. 2 (кривая 1). По достижении необходимого значения температуры проводилось термостатирование образца при практически неизменной величине плотности тока. Протекание тока при АПС вызывает выделение теплоты Джоуля и повышение температуры в токопроводящих частях установки и в образце (рис. 2, кривые 2,3).

В качестве граничного условия для торцевой поверхности верхнего стального электрода задавалась временна́я зависимость действующего значения плотности тока, обеспечивающего разогрев нижней поверхности образца до температуры $730 \mathrm{~K}$. На торце нижнего электрода задавался нулевой потенциал. Водяное охлаждение на торцах электродов описывалось при помощи уравнения Ньютона для конвективного теплового потока. С боковой поверхности установки осуществлялся радиационный теплоотвод в окружающую среду, описываемый уравнением Стефана-Больцмана.
Процесс спекания был рассмотрен для образцов однородного состава и составных, содержащих слои материалов с отличающимися диапазонами рабочих температур и температур спекания. Геометрические размеры образцов были приняты одинаковыми. Образцы представляли собой цилиндры диаметром 20 мм и высотой 2 мм. Однородный образец состоял из теллурида висмута $\left(\mathrm{Bi}_{2} \mathrm{Te}_{3}\right)$. Составные образцы содержали слои одинаковой толщины, различающиеся химическим составом. Двухслойный образец состоял из теллурида висмута и теллурида свинца (PbTe). Трехслойный образец состоял из слоев $\mathrm{Bi}_{2} \mathrm{Te}_{3}$, PbTе и антимонида кобальта $\mathrm{CoSb}_{3}$.

Слой диоксида кремния, введенный в оснастку между матрицей пресс-формы и верхним пуансоном и образцом, позволяет направить большую часть тока через верхний пуансон, тем самым разогревая его до большей температуры. Это приводит к росту разницы температуры в объеме образца (рис. 3). Результаты моделирования показывают, что увеличенное значение $\Delta T_{z}$ при спекании

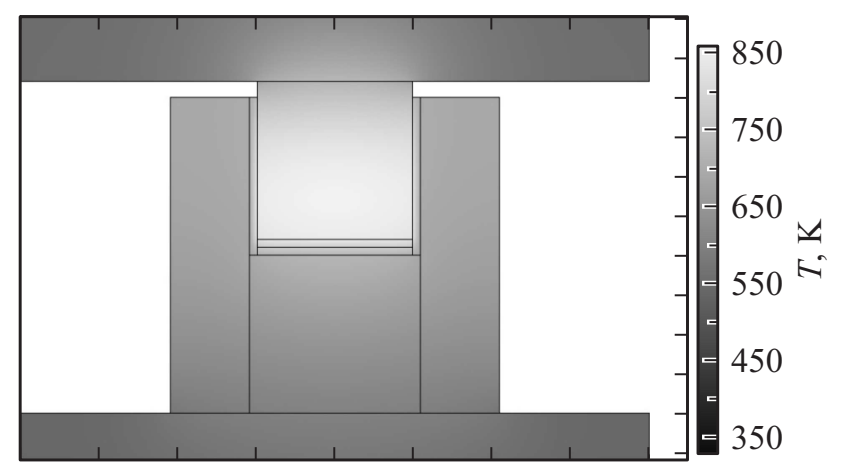

Рис. 3. Распределение температуры в деталях установки АПС и составном образце $\mathrm{Bi}_{2} \mathrm{Te}_{3} / \mathrm{PbTe}$ при спекании в оснастке с изоляционным слоем толщиной 1 мм.

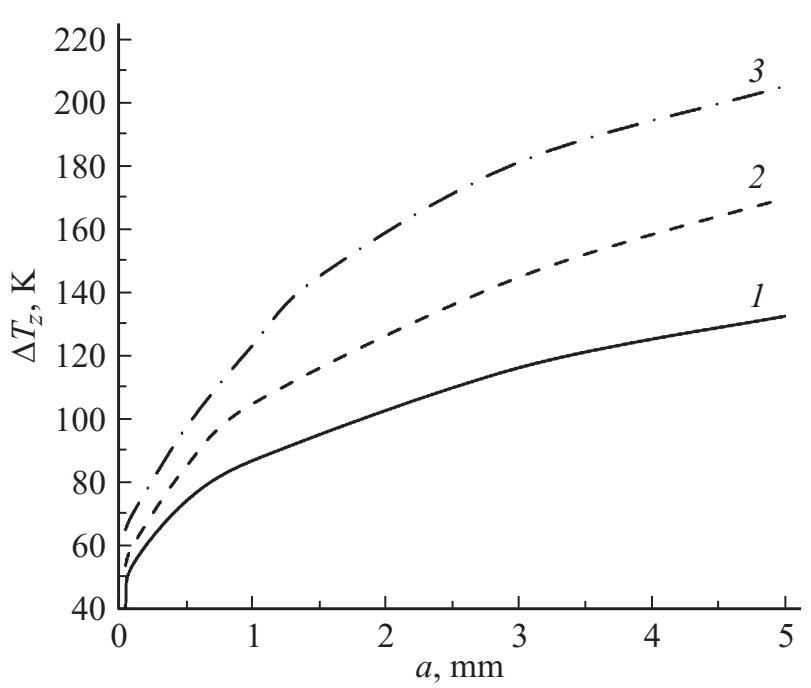

Рис. 4. Зависимости перепада температуры по высоте образца от толщины изоляционного слоя $a$ для образцов различного состава: $1-$ однородный $\mathrm{Bi}_{2} \mathrm{Te}_{3} ; 2-$ двухслойный составной $\mathrm{Bi}_{2} \mathrm{Te}_{3} / \mathrm{PbTe} ; 3$ - трехслойный составной $\mathrm{Bi}_{2} \mathrm{Te}_{3} / \mathrm{PbTe} / \mathrm{CoSb}_{3}$. 


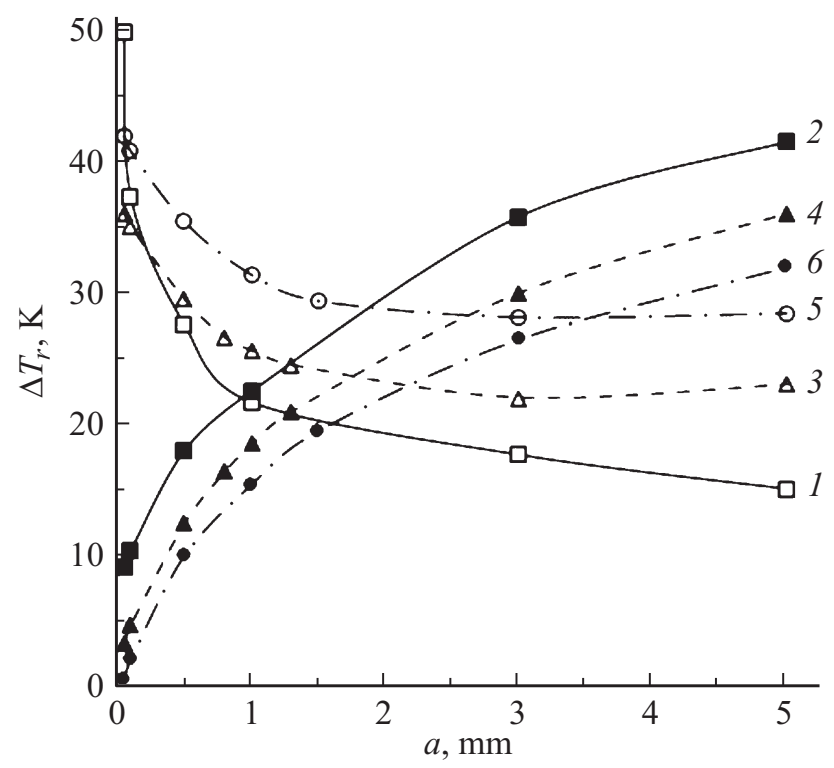

Рис. 5. Зависимости перепада температур в радиальном направлении на верхней $(1,3,5)$ и нижней $(2,4,6)$ границах образца. Образцы: 1,2 - однородный $\mathrm{Bi}_{2} \mathrm{Te}_{3} ; 3,4$ - двухслойный составной $\mathrm{Bi}_{2} \mathrm{Te}_{3} / \mathrm{PbTe} ; 5,6$ - трехслойный составной $\mathrm{Bi}_{2} \mathrm{Te}_{3} / \mathrm{PbTe} / \mathrm{CoSb}_{3}$.

сохраняется в течение 5-8 мин (рис. 2, кривые 2, 3), что соответствует стандартной продолжительности процесса АПС при максимальном токе.

Величина температурного перепада между верхней и нижней границами образца $\Delta T_{z}$ зависит от толщины изоляционного слоя $a$ и материала образца (рис. 4). Увеличение толщины изоляционного слоя сопровождается изменением радиального перепада температуры в образце $\Delta T_{r}$. Максимальных значений перепад температур в радиальном направлении $\Delta T_{r}$ достигает на верхней и нижней границах образца. На рис. 5 приведены зависимости $\Delta T_{r}$ от толщины изоляционного слоя $а$ для образцов различного состава. Величина $\Delta T_{r}$ на нижней поверхности образца растет с увеличением $a$ и уменьшается на верхней поверхности. Оптимальное значение толщины изоляции $a_{\text {opt }}$ соответствует, по-видимому, точке пересечения кривых, где значения максимальных $\Delta T_{r}$ в процессе спекания примерно одинаковы. Для однородного образца с рассмотренными геометрическими размерами это значение составило 1 мм. Для составных образцов оптимальная толщина изоляции оставила 1.8 и $3.4 \mathrm{MM}$ для $\mathrm{Bi}_{2} \mathrm{Te}_{3} / \mathrm{PbTe}$ и $\mathrm{Bi}_{2} \mathrm{Te}_{3} / \mathrm{PbTe} / \mathrm{CoSb}_{3}$ соответственно.

Таким образом, в работе показана возможность проведения АПС образцов термоэлектриков в градиентном температурном поле с $\Delta T_{z}$, достигающем нескольких сотен градусов, с использованием модифицированной оснастки, содержащей слой электрической изоляции.

Величина $\Delta T_{r}$ может достигать $35-50 \mathrm{~K}$ при малых $(0.05 \mathrm{Mм})$ и $32-42 \mathrm{~K}$ при больших (5 мм) величинах $a$. При использовании изоляции толщиной, равной $a_{\text {opt }}(1-3.5 \mathrm{Mм})$, величина $\Delta T_{r}$ может быть понижена до $22-27 \mathrm{~K}$ для образцов различного состава. Увеличе- ние диапазона температур спекания материалов составных образцов подразумевает рост $a_{\text {opt }}$.

Предложенный метод может быть применен для спекания образцов с геометрическими размерами и составом, отличными от рассмотренных в работе. Однако значения $\Delta T_{r}, \Delta T_{z}$ и $a_{\text {орt }}$ могут отличаться от представленных в работе и требуют дополнительных расчетов.

Работа выполнена при государственной финансовой поддержке ведущих университетов Российской Федерации (субсидия 074-U01).

\section{Список литературы}

[1] D.M. Rowe. Thermoelectrics Handbook: Macro to Nano, ed. by D.M. Rowe (CRC Press, N.Y.-London-Tokyo, 2006) p. $1-1$.

[2] A. Saramat, G. Svensson, A.E.C. Palmqvist, C. Stiewe, E. Mueller, D. Platzek, S. G.K. Williams, D.M. Rowe, J.D. Bryan, G.D. Stucky. J. Appl. Phys., 99, 023708 (2006).

[3] V.L. Kuznetsov, P.P. Edwards. ChemSusChem., 3 (1), 44 (2010).

[4] Л.И. Анатычук, Л.Н. Вихор. Сб. тр. конф. „Термоэлектрики и их применения“" (Санкт-Петербург, 2013) с. 434.

[5] S. Yoon, J. Cho, H. Koo, S. Bae, S. Ahn, G.R. Kim, J.S. Kim, C. Park. J. Electron. Mater., 43 (2), 414 (2014).

[6] O. Guillon, J. Gonzalez-Julian, B. Dargatz, T. Kessel, G. Schierning, J. Räthel, M. Herrmann. Adv. Eng. Mater., 16 (7), 830 (2014).

[7] M. Suárez, A. Fernández, J.L. Menéndez, R. Torrecillas, H.U. Kessel, J. Hennicke, R. Kirchner, T. Kessel. Sintering applications (Intech, 2013) p. 319.

[8] L.P. Bulat, I.A. Drabkin, V.V. Karatayev, V.B. Osvenskii, Yu.N. Parkhomenko, M.G. Lavrentev, A.I. Sorokin, D.A. Pshenai-Severin, V.D. Blank, G.I. Pivovarov, V.T. Bublik, N.Yu. Tabachkova. J. Electron. Mater., 42 (7), 2110 (2013).

[9] I.A. Drabkin, V.B. Osvenskii, Yu.N. Parkhomenko, A.I. Sorokin, G.I. Pivovarov, L.P. Bulat. J. Thermoelectricity, 3, 35 (2013).

[10] Л.П. Булат, И.А. Драбкин, А.В. Новотельнова, В.Б. Освенский, Ю.Н. Пархоменко, А.И. Сорокин, Д.А. ПшенайСеверин, И.А. Нефедова. Письма ЖТФ, 40 (21), 79 (2014).

Редактор Л.В. Шаронова

\section{The simulation of field activated thermoelectric materials sintering}

L.P. Bulat ${ }^{1}$, A.V. Novotelnova ${ }^{1}$, V.B. Osvenskii ${ }^{2}$, A.S. Tukmakova ${ }^{1}$, D. Yerezhep ${ }^{1}$

1 ITMO University, 197101 St. Petersburg, Russia

2 „Giredmet" Ltd., 119017 Moscow, Russia

Abstract We have created a time-dependent computer model of segmented nanothermoelectrics field activated sintering. The tooling modification containing electric isolating layer has been proposed. This tooling leads to a temperature difference formation up to hundreds of degrees in a sample. A possibility to reduce a radial temperature gradient has been shown. 